\title{
« Le patrimoine industriel de la chimie », Patrimoine industriel. Archéologie, technique, mémoire
}

CILAC, $n^{\circ}$ 69, décembre 2016

\section{Marina Gasnier}

\section{(Q) OpenEdition \\ Journals}

\section{Édition électronique}

URL : http://journals.openedition.org/artefact/1837

DOI : $10.4000 /$ artefact. 1837

ISSN : 2606-9245

\section{Éditeur :}

Association Artefact. Techniques histoire et sciences humaines, Presses universitaires du Midi

\section{Édition imprimée}

Date de publication : 30 mai 2018

Pagination : 318-322

ISBN : 978-2-7535-7494-6

ISSN : 2273-0753

\section{Référence électronique}

Marina Gasnier, « « Le patrimoine industriel de la chimie », Patrimoine industriel. Archéologie, technique, mémoire », Artefact [En ligne], 7| 2017, mis en ligne le 14 février 2019, consulté le 23 septembre 2020. URL : http://journals.openedition.org/artefact/1837 ; DOI : https://doi.org/10.4000/artefact.1837

Ce document a été généré automatiquement le 23 septembre 2020.

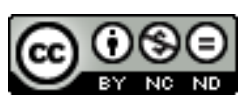

Artefact, Techniques, histoire et sciences humaines est mise à disposition selon les termes de la Licence Creative Commons Attribution - Pas d'Utilisation Commerciale - Pas de Modification 4.0 International. 


\title{
« Le patrimoine industriel de la chimie », Patrimoine industriel. Archéologie, technique, mémoire
}

CILAC, $n^{\circ}$ 69, décembre 2016

\author{
Marina Gasnier
}

\section{RÉFÉRENCE}

« Le patrimoine industriel de la chimie », Patrimoine industriel. Archéologie, technique, mémoire, CILAC, $\mathrm{n}^{\circ}$ 69, décembre 2016.

Dans le prolongement de l'Année internationale de la chimie organisée en 2011 par l'IUPAC et l'Unesco, la revue Patrimoine industriel dédie un numéro spécial à la thématique du patrimoine industriel de ce secteur, par ailleurs objet d'une enquête lancée en 2016 par l'Heritage Council Europe (P. Bret et al., p. 6). Associée depuis les années 1960 à des sinistres sanitaires ou cause de multiples nuisances environnementales, l'industrie de la chimie a pourtant longtemps été symbole de progrès et d'innovation. C'est donc dans une volonté partagée de "réhabiliter " symboliquement et matériellement le patrimoine issu de ce secteur d'activité que s'inscrit ce numéro collectif dirigé par Patrice Bret. Mené en collaboration avec l'association Valpasti et le Groupe d'histoire de la chimie, ce dossier a bénéficié du mécénat de Solvay. Il est composé de trois parties dont la première pose le contexte de l'étude, sa motivation, ainsi qu'une perspective historique. Constituant les deux tiers du volume, la deuxième partie présente des études de cas assorties de quelques focus. Enfin, les dernières pages posent la question des archives et des collections conservées en France. Forte de sa nouvelle mise en page, la revue Patrimoine industriel nous livre un dossier tout à fait intéressant à plusieurs égards.

2 À juste titre, d'emblée est soulignée la faible prise en compte du patrimoine industriel de la chimie en France et sa fragilité face à l'innovation et à la désindustrialisation. Ce 
constat place les initiateurs du dossier dans une perspective de sensibilisation des entreprises à la sauvegarde de leur héritage afin de témoigner de sa diversité. C'est en ce sens qu'est présentée la douzaine de cas d'études qui ne saurait toutefois être considérée comme un premier inventaire du patrimoine industriel de la chimie en France (P. Bret et al., p. 15). Ou alors ce serait nier le travail remarquable d'ores et déjà mené depuis des années par les anciens services de l'Inventaire général en régions. Riche d'environ 600 sites industriels identifiés sous l'intitulé «usine de produits chimiques ", ce corpus ne peut être ignoré dans le cadre d'une telle étude car il est le fruit d'enquêtes de terrain et d'un dépouillement archivistique systématique contribuant à enrichir la connaissance. Si les contours des résultats de ce premier repérage peuvent être nuancés, voire approfondis selon l'orientation de l'étude envisagée, il reste une base de travail tout à fait incontournable. En témoigne, l'un des cas d'étude du dossier portant sur la parfumerie (G. Benalloul et G. Buffa). Identifiées lors d'un inventaire exhaustif mené par la ville de Grasse en partenariat avec le service Patrimoine, Traditions, Inventaire de la Région Provence-Alpes-Côte d'Azur, les constructions liées à l'histoire de la parfumerie subsistant dans le tissu urbain se révèlent être un objet d'étude extrêmement intéressant pour expliquer la transformation d'une ancienne cité médiévale en capitale mondiale du parfum à compter de la fin du XVIII ${ }^{e}$ siècle. L'article montre l'intérêt de l'inventaire exhaustif comme phase préalable à toute étude ambitionnant une analyse relative à la mutation du bâti, à l'évolution des techniques, ou encore aux transformations économiques et sociales. À Grasse, comme dans bien des territoires étudiés dans le dossier, les ateliers implantés dans le tissu urbain se sont vidés de leur substance compte tenu du choix des industriels d'investir des espaces plus vastes en périphérie de la ville et mieux adaptés aux nouveaux process. Face à cette relocalisation, se pose la question de la gestion de ce patrimoine industriel laissé vacant dans le cadre des politiques urbaines (ibid., p. 29). S'il est difficile d'apporter des éléments de réponse précis s'agissant avant tout de situations singulières et de choix politiques et culturels locaux, ce type de questionnement est particulièrement saillant pour le patrimoine industriel et peut, toutefois, profiter des éclairages fournis par la recherche. En ce sens, la perspective affichée de ce numéro collectif de poursuivre les études sur le patrimoine des installations liées à la chimie ne peut être qu'encouragée dans le but d'une meilleure connaissance et préservation. Car s'il est présenté ici comme «invisible» (P. Bret et al., p. 7-8), ce patrimoine, doté de surfaces et d'espaces bâtis tout en contrastes, semble surtout souffrir d'une absence de reconnaissance. Nous avons effectivement affaire à des objets encore trop souvent considérés en dehors du champ du patrimoine culturel. Et accéder à leur reconnaissance est d'autant plus ardu qu'il s'agit d'un secteur particulièrement frappé par la rapidité de l'obsolescence des techniques, des procédés et des équipements soumis aux destructions. Même glorieux, le passé lié à l'industrie de la chimie doit s'effacer devant l'innovation. C'est pourquoi les industriels du secteur sont bien souvent restés à l'écart des enjeux de la patrimonialisation (P. Bret et al., p. 9). Aussi, à l'instar de tout site historique pérennisant son activité - dans le cadre d'une réhabilitation ou non - la difficulté réside dans l'équilibre à trouver pour concilier la préservation patrimoniale et les politiques d'innovation développées par les entreprises. La gageure n'est pas impossible. Elle implique une réflexion sur la notion de « patrimoine en mouvement », bien que celle-ci ne soit pas posée en ces termes dans le dossier. 
3 Malgré une approche partielle de la thématique justifiée par Florence Hachez-Leroy en propos introductif, l'un des intérêts de ce numéro réside dans la qualité des articles donnant une variété de regards complémentaires de l'histoire de l'industrie chimique française et, de fait, de la diversité de ses traces dans le paysage. Tout en replaçant avec justesse cette branche sectorielle dans une perspective historique, Denis Woronoff souligne combien ce patrimoine compte parmi les plus complexes de notre héritage industriel en raison d'une architecture "sans toit ni mur " (D. Woronoff, p. 18) et des nœuds techniques dont témoignent les liaisons entre les raffineries et les lieux de production. Cet emboitement d'échelles, déjà observé lors de l'avancée de certains travaux portant sur le patrimoine industriel, prend encore une autre envergure avec le secteur de la chimie. Il est véritablement question d'une géographie industrielle de la chimie dont l'angle d'attaque peut s'inscrire à d'autres niveaux que celui des flux. En atteste, par exemple, la politique d'implantation multi-site à l'échelle internationale des soudières Solvay à l'origine de la construction d'une vingtaine d'usines à travers le monde, entre 1865 et 1918, et de ses structures sociales connexes (Ph. Mioche). Or, l'une des richesses du dossier tient dans le fait qu'il n'est pas seulement question de lieux de production, mais bien d'un système complexe aux ramifications variées comme le rappelle Erik Langlinay en écho aux propos de Pierre Lamard et Nicolas Stoskopf (p. 138). Cette approche systémique implique d'interroger ce que recouvre l'industrie chimique pour bien cerner le propos. Plusieurs articles du dossier renvoient ainsi aux écoles de chimie, aux organisations patronales, ainsi qu'aux contextes économiques et politiques. C'est en ce sens que Denis Woronoff, tout en rappelant son caractère empirique jusqu'au dernier tiers du XvIII ${ }^{\mathrm{e}}$ siècle, montre bien l'importance du lien qui s'instaure au siècle suivant entre la formation au sein des écoles de chimie, les entreprises et la présence de laboratoires à proximité des lieux de production. Il est d'ailleurs tout à fait remarquable de constater que cette configuration caractéristique de la modernité et de l'originalité de l'industrie chimique, en prise avec son territoire et ses ressources en présence, s'observe aujourd'hui dans le cadre de la réhabilitation de sites industriels ancestraux riches de savoir-faire spécifiques. Prônée par la Stratégie de Lisbonne, cette trilogie alliant formation, recherche et lieux de production a donc des racines profondes qui ne peuvent être ignorées au regard de l'Histoire. Indirectement, l'actualité est ainsi au cœur du propos. De la même manière, l'internationalisation - regardée aujourd'hui par certains avec circonspections'illustre avec le groupe belge Solvay dont l'esprit procède, non seulement d'une dynamique entrepreneuriale, mais aussi d'une politique paternaliste très ambitieuse que nous fait découvrir Philippe Mioche et qui témoigne d'une vision sociétale très intéressante. Malgré une implantation des usines au-delà des frontières, on remarque une volonté d'unité reposant sur des conceptions techniques, architecturales et sociales similaires d'un pays à l'autre (cités ouvrières, logements pour les cadres, écoles, centres de soins, lieux de jeu avec les casinos), bien qu'adaptées aux situations géographiques et à la culture locale. La question environnementale n'est pas plus ignorée. Développée dès le début $d u$ xIx ${ }^{e}$ siècle en Provence, l'industrie de la soude alimentant les verreries et les savonneries - à l'instar de Solvay avant une diversification de sa production -, pose avec force le problème de la pollution subsistant au-delà de la préservation même du bâti (X. Daumalin). Toutefois, ce dossier montre aussi que cette problématique ne saurait s'inscrire sous le seul angle culpabilisant. En effet, au sujet de la carbonisation industrielle $\mathrm{du}$ bois et de la récupération des produits pyroligneux, Jean-Philippe Passaqui évoque un autre rapport à l'environnement qui est davantage celui d'une 
interdépendance avec les activités de l'homme. Il souligne les liens étroits entretenus entre l'exploitation des ressources et la gestion des forêts après la fin de la sidérurgie au bois, et interroge en ce sens la question de la reconversion pour le bois des forêts (p.117). Mais bien entendu, les procédés liés à la chimie induisent d'autres risques que ceux de l'empreinte environnementale. L'éloignement de certains sites des tissus urbains rend compte de la dangerosité de leurs productions dont témoigne le secteur des poudres et des explosifs représenté par plus d'une vingtaine de sites en France, caractéristique des modes de production successifs et dont la moitié est datée du XVII ${ }^{\mathrm{e}}$ siècle (P. Bret). L'étude met en lumière une architecture particulière constituée de bâtiments isolés les uns des autres, séparés par de larges murs ou des merlons afin d'atténuer le souffle en cas d'explosion. La structure même du bâti est impactée par la nature de l'activité. Les murs sont épais tandis que la couverture est légère pour dégager le souffle. Ce lien étroit entre l'enveloppe architecturale et le type de production qu'elle abrite est souligné dans plusieurs études de cas du dossier, révélant ainsi, non seulement une diversité typologique extrêmement riche témoignant de l'histoire des installations liées à l'industrie de la chimie, mais aussi une évolution des procédés techniques adoptés. Par exemple, à partir du milieu du XIX ${ }^{e}$ siècle à Grasse, l'adaptation du bâti à l'évolution des technologies est parfaitement identifiable, notamment par la modernisation des procédés de distillation recourant à la vapeur et entrainant, dans le même temps, la construction d'édifices de taille plus importante que les traditionnels usines-immeubles grassois, ainsi qu'une ventilation assurée par de vastes baies garnies de transennes (G. Benalloul et G. Buffa, p. 33).

Mais force est de constater au fil des pages, que le patrimoine industriel de la chimie compte parmi les plus menacés en raison d'une disparition importante de son bâti et encore davantage de ses équipements techniques. En dehors de la préservation de certains moulins à huile, la parfumerie à Grasse en témoigne, ainsi que l'industrie des allumettes chimiques (P.Smith), ou encore l'industrie rhumière à La Réunion (X. Le Terrier). Ce numéro spécial atteste autant de la pauvreté du patrimoine archivistique dont une grande partie a été perdue. Comme le précise Érik Langlinay, les industriels sont peu enclins à se mobiliser en faveur de la sauvegarde de leurs archives lors d'une cessation d'activité et d'une perte des emplois. Toutefois, au-delà des fonds privés, le travail mené par Hervé Joly sur l'entreprise Gillet montre qu'il existe d'autres voies pour retracer l'histoire de l'industrie chimique française, parmi lesquelles les archives d'État, les sources des administrations locales, des écoles de chimie, ou encore les archives syndicales (E. Langlinay, p. 142). Il ne faudrait pas non plus négliger la mémoire orale des anciens salariés qu'il devient urgent de collecter, pas plus que d'autres travaux récents témoignant de la richesse de fonds particuliers dont celui de l'Institut du pin sur la chimie des résines en Aquitaine (voir les travaux de Marcin Krasnodebski).

5 Dans ce sillage et à quelques exceptions près, la muséographie française accorde très peu de place à la chimie. Celle-ci est davantage présentée par du matériel de laboratoire plutôt que par des procédés de fabrication. Enfin, et comme c'est souvent le cas en matière de défense du patrimoine, ce numéro spécial témoigne de la mobilisation importante du milieu associatif (F. Buisson, p. 96 ; Ph. Matin, p. 77) et révèle la difficile patrimonialisation de cet héritage compte tenu de son envergure, de sa spécificité, et de son image fortement dégradée depuis la fin du $\mathrm{xx}^{\mathrm{e}}$ siècle. Mais, du textile aux engrais en passant par la pharmaceutique, la plasturgie ou encore la pétrochimie, ce sont aussi 
des volumes et des espaces hors normes qui rendent complexe la double question de leur intégration dans le paysage et de leur reconversion. Le cas des raffineries de pétrole (N. Rafowicz) est particulièrement évocateur. Et il est important de le considérer car il est aussi annonciateur des futurs débats relatifs aux enjeux de la patrimonialisation des centrales nucléaires, dans un contexte de fermeture progressive d'une grande partie de ces dernières à l'horizon 2050.

\section{AUTEUR}

\section{MARINA GASNIER}

Université Bourgogne Franche-Comté, FEMTO-ST/RECITS (UMR 6174) 